\title{
Deterministic Stabilization of a Dynamical System using a Computational Approach
}

\author{
Isobeye George ${ }^{1}$, Jeremiah U. Atsu ${ }^{2}$, Enu-Obari N. Ekaka-a ${ }^{3}$ \\ ${ }^{1}$ Department of Mathematics/Statistics, Ignatius Ajuru University of Education, PMB 5047, Port Harcourt, Nigeria, \\ ${ }^{2}$ Department of Mathematics/Statistics, Cross River University of Technology, Calabar, Nigeria, \\ ${ }^{3}$ Department of Mathematics, Rivers State University, Port Harcourt, Nigeria.
}

\begin{abstract}
The qualitative behavior of a multi-parameter dynamical system has been investigated. It is shown that changes in the initial data of a dynamical system will affect the stabilization of the steady-state solution which is originally unstable. It is further shown that the stabilization of a five-dimensional dynamical system can be used as an alternative method of verifying qualitatively the concept of the stability of a unique positive steady-state solution. These novel contributions have not been seen elsewhere; these are presented and discussed in this paper.
\end{abstract}

Keywords - Deterministic, stabilization, dynamical system, steady-state solution, changing initial data.

\section{INTRODUCTION}

Agarwal and Devi (2011) studied in detail the mathematical analysis of a resource-dependent competition model using the method of local stability analysis. Other relevant mathematical approaches to the concept of stability analysis have been done [Rescigno (1977);Hallam, Clark and Jordan (1983);Hallam, Clark and Lassiter (1983);Hallam and Luna (1984);Freedman and So (1985); Lancaster and Tismenetssky (1985);De Luna and Hallam (1987); Zhien and Hallam (1987);Freeman and Shukla (1991);Huaping and Zhien (1991);Garcia-Montiel and Scatena (1994); Chattopadhyay (1996);Hsu and Waltman (1998); Dubey and Hussain (2000);Hsu, Li and Waltman (2000);Thieme (2000); Shukla, Agarwal, Dubey and Sinha (2001); Ekaka-a (2009); Shukla, Sharma, Dubey and Sinha (2009); Yan and Ekaka-a (2011);Dhar, Chaudhary and Sahu (2013); Akpodee and Ekaka-a (2015)]. The method of thispresent study uses the technique of a numerical simulation to quantify the qualitative characteristics of a complex dynamical system with changing initial data.

\section{MATHEMATICAL FORMULATION}

We have considered the following continuous multiparameter system of nonlinear first order ordinary differential equations indexed by the appropriate initial conditions according to Agarwal and Devi (2011):

$$
\begin{aligned}
& \frac{d x_{1}}{d t}=a_{1} x_{1}-a_{2} x_{1}^{2}-\alpha x_{1} x_{2}+\alpha_{1} x_{1} R-k_{1} \delta_{1} x_{1} T, x_{1}(0)= \\
& x_{10} \geq 0, \quad \text { (1a) } \\
& \frac{d x_{2}}{d t}=b_{1} x_{2}-b_{2} x_{2}^{2}-\beta x_{1} x_{2}+\beta_{1} x_{2} R-k_{2} \delta_{2} x_{2} T, x_{2}(0)= \\
& x_{20} \geq 0, \quad(1 \mathrm{~b}) \\
& \frac{d R}{d t}=c_{1} R-c_{2} R^{2}-\alpha_{1} x_{1} R-\beta_{1} x_{2} R-k \gamma R T, R(0)=R_{0} \geq \\
& 0, \quad(1 \mathrm{c}) \\
& \frac{d P}{d t}=\eta x_{1}+\eta x_{2}-\left(\lambda_{0}+\theta\right) P, P(0)=P_{0} \geq 0, \\
& \frac{d T}{d t}=Q+\mu \theta P-\delta_{0} T-\delta_{1} x_{1} T-\delta_{2} x_{2} T-\gamma R T, \\
& T_{0} \geq 0, \quad \quad \quad(1 \mathrm{e})
\end{aligned}
$$

where

$x_{1}$ and $x_{2}$ are the densities of the first and second competing species, respectively, $R$ is the density of resource biomass, $P$ is the cumulative concentration of precursors produced by species forming the toxicant, $T$ is the concentration of the same toxicant in the environment under consideration, $Q$ is the cumulative rate of emission of the same toxicant into the environment from various external sources, $a_{1}$ and $b_{1}$ are the intrinsic growth rates of the first and second species, respectively, $a_{2}$ and $b_{2}$ are intraspecific interference coefficients, $\alpha, \beta$ are the interspecific interference coefficients of first and second species, respectively, $\alpha_{1}$ and $\beta_{1}$ are the growth rate coefficients of first and second species, respectively due to resource biomass. $k_{1}, k_{2}$ and $k$ are fractions of the assimilated amount directly affecting the growth rates of densities of competing species and resource biomass, $\eta$ is the growth rate coefficient of the cumulative concentration of precursors. $\lambda_{0}$ is its depletion rate coefficient due to natural factors whereas $\theta$ is the depletion rate coefficient caused by its transformation into the same toxicant of concentration $T . \mu$ is the rate of the formation of the toxicant from precursors of competing species. $\delta_{1}, \delta_{2}$ and $\gamma$ are the rates of depletion of toxicant in the environment due to uptake of toxicant by species and their resource biomass, respectively.

It is assumed that the resource biomass grows logistically with the supply rate of the external resource input to the system by constant $c_{1}$ and its density reduces due to certain

Page | 24 
degradation factors present in the environment at a rate $c_{2}$.It is further assumed that the toxicant in the environment is washed out or broken down with rate $\delta_{0}$.

\section{Research Question}

For the purpose of this study, we have considered the following vital research question:How does a given dynamical multi-parameter system of continuous nonlinear first-order ordinary differential equations respond to a qualitative characteristic, that is, assuming a point $\left(x_{1 e}, x_{2 e}, R_{e}, P_{e}, T_{e}\right)$ is an arbitrary steady-state solution, as the independent variable $t$ approaches infinity $(t \rightarrow \infty)$, do $x_{1} \rightarrow x_{1 e}, x_{2} \rightarrow x_{2 e}, R \rightarrow R_{e}, P \rightarrow P_{e}, T \rightarrow T_{e}$ under some simplifying initial conditions? This mathematical idea is a necessary and sufficient condition that quantifies the concept of the stabilization of the steady-state solution $\left(x_{1 e}, x_{2 e}, R_{e}, P_{e}, T_{e}\right)$ (Yan and Ekaka-a, 2011). In other words, for a complex system of nonlinear first-order ordinary differential equations whose interacting functions are continuous and partially differentiable, what is the likely qualitative characteristic of such a system? The focus of this chapter will tackle the following proposed problem that is clearly defined next.

\section{Research Hypothesis}

It is a well-established ecological fact that the initial ecological data, which mathematicians called initial conditions, are not static characteristic values of a dynamical system. The corresponding core research question is, when the initial data change, how does the dynamical system respond to this change over a longer period of time? This hypothesis if successfully tested and proved in this research, has the potential to provide an insight in the further study of ecosystem stability and ecosystem planning.

\section{Method of Analysis}

A well-defined MATLAB ODE45 function has been used to construct tables to determine the effect of changing values of initial data on the stability of the dynamical system for large values of the independent variable $t$. Following Agarwal and Devi (2011), the values of parameter values which are used in the numerical simulations for system (1) are:

$a_{1}=5, \quad a_{2}=0.22, \quad \alpha=0.007, \quad \alpha_{1}=0.2, \quad k_{1}=0.1$, $\delta_{1}=0.05, \quad b_{1}=3, \quad b_{2}=0.26, \quad \beta=0.008, \quad \beta_{1}=$ $0.04, \quad k_{2}=0.2, \quad \delta_{2}=0.04, \quad \eta=0.5, \quad \lambda_{0}=0.01$, $\theta=3, \quad \mu=0.2, \quad \delta_{0}=7, \quad \gamma=0.3, \quad c_{1}=10$, $c_{2}=0.3, \quad k=0.1, \quad Q=30$.

\section{RESULTS AND DISCUSSIONS}

Some twenty (20) numerical simulations are observed to determine the effect of changing values of initial data on the stability of the dynamical system for $t=3650$ days as shown in Table 1 below:

Table.1: Numerical simulation of a dynamical system for changing initial data at $t=3650$ days, using a MATLAB ODE45 numerical scheme.

\begin{tabular}{|c|c|c|c|c|c|c|c|}
\hline Example & Initial Data (ID) & $\begin{array}{c}\text { Independent } \\
\text { Variable t days }\end{array}$ & $\mathrm{x}_{1 \mathrm{e}}$ & $\mathrm{x}_{2 \mathrm{e}}$ & $\mathrm{R}_{\mathrm{e}}$ & $\mathrm{P}_{\mathrm{e}}$ & $\mathrm{T}_{\mathrm{e}}$ \\
\hline 1 & 1 & 3650 & 25.4443 & 15.2308 & 30.7270 & 6.7195 & 2.1454 \\
\hline 2 & 2 & 3650 & 25.3091 & 15.2308 & 30.6851 & 6.7195 & 2.1086 \\
\hline 3 & 3 & 3650 & 25.3551 & 15.2308 & 30.6872 & 6.7195 & 2.1054 \\
\hline 4 & 4 & 3650 & 25.3783 & 15.2308 & 30.6901 & 6.7195 & 2.1042 \\
\hline 5 & 5 & 3650 & 25.3923 & 15.2308 & 30.6931 & 6.7195 & 2.1041 \\
\hline 6 & 6 & 3650 & 25.4018 & 15.2308 & 30.6958 & 6.7195 & 2.1046 \\
\hline 7 & 7 & 3650 & 25.4085 & 15.2308 & 30.6983 & 6.7195 & 2.1055 \\
\hline 8 & 8 & 3650 & 25.4129 & 15.2308 & 30.6979 & 6.7195 & 2.1066 \\
\hline 9 & 9 & 3650 & 25.4169 & 15.2308 & 30.6997 & 6.7195 & 2.1080 \\
\hline 10 & 10 & 3650 & 25.4202 & 15.2308 & 30.7014 & 6.7195 & 2.1094 \\
\hline 11 & 11 & 3650 & 25.4229 & 15.2308 & 30.7030 & 6.7195 & 2.1108 \\
\hline 12 & 12 & 3650 & 25.4252 & 15.2308 & 30.7045 & 6.7195 & 2.1123 \\
\hline 13 & 13 & 3650 & 25.4272 & 15.2308 & 30.7058 & 6.7195 & 2.1138 \\
\hline 14 & 14 & 3650 & 25.4288 & 15.2308 & 30.7071 & 6.7195 & 2.1153 \\
\hline 15 & 15 & 3650 & 25.4303 & 15.2308 & 30.7083 & 6.7195 & 2.1168 \\
\hline 16 & 16 & 3650 & 25.4316 & 15.2308 & 30.7094 & 6.7195 & 2.1183 \\
\hline
\end{tabular}




\begin{tabular}{|c|c|c|c|c|c|c|c|}
\hline Example & Initial Data (ID) & $\begin{array}{c}\text { Independent } \\
\text { Variable t days }\end{array}$ & $\mathrm{x}_{1 \mathrm{e}}$ & $\mathrm{x}_{2 \mathrm{e}}$ & $\mathrm{R}_{\mathrm{e}}$ & $\mathrm{P}_{\mathrm{e}}$ & $\mathrm{T}_{\mathrm{e}}$ \\
\hline 17 & 17 & 3650 & 25.4328 & 15.2308 & 30.7105 & 6.7195 & 2.1197 \\
\hline 18 & 18 & 3650 & 25.4338 & 15.2308 & 30.7115 & 6.7195 & 2.1211 \\
\hline 19 & 19 & 3650 & 25.4347 & 15.2308 & 30.7125 & 6.7195 & 2.1225 \\
\hline 20 & 20 & 3650 & 25.4356 & 15.2308 & 30.7134 & 6.7195 & 2.1239 \\
\hline
\end{tabular}

where

ID $1=(2,0.01,0.01,0.1,0.1)$, ID $2=(0.10,0.01,0.01,0.1$,

$0.1)$,

ID $3=(0.15,0.01,0.01,0.1,0.1)$, ID $4=(0.20,0.01,0.01$, $0.1,0.1)$,

ID $5=(0.25,0.01,0.01,0.1,0.1)$, ID $6=(0.30,0.01,0.01$, $0.1,0.1)$,

ID $7=(0.35,0.01,0.01,0.1,0.1)$, ID $8=(0.40,0.01,0.01$, $0.1,0.1)$

ID $9=(0.45,0.01,0.01,0.1,0.1)$, ID $10=(0.50,0.01,0.01$, $0.1,0.1)$

ID $11=(0.55,0.01,0.01,0.1,0.1)$, ID $12=(0.60,0.01$, $0.01,0.1,0.1)$,

ID $13=(0.65,0.01,0.01,0.1,0.1)$, ID $14=(0.70,0.01$, $0.01,0.1,0.1)$,
ID $15=(0.75,0.01,0.01,0.1,0.1)$, ID $16=(0.80,0.01$, $0.01,0.1,0.1)$,

ID $17=(0.85,0.01,0.01,0.1,0.1)$, ID $18=(0.90,0.01$, $0.01,0.1,0.1)$,

ID $19=(0.95,0.01,0.01,0.1,0.1)$, ID $20=(1,0.01,0.01$, $0.1,0.1)$.

Considering Table 1, we deduced mathematically that, as $t \rightarrow \infty$ for the given initial conditions, $x_{1} \rightarrow x_{1 e}, x_{2} \rightarrow x_{2 e}$, $R \rightarrow R_{e}, P \rightarrow P_{e}, T \rightarrow T_{e}$. We have shown that as the initial data are changing, the system is approaching its steadystate. This shows that changes in the initial data of a dynamical system will affect the stabilization of the steadystate solution which is originally unstable.

Table.2: Test of stability of steady-state solutions for changing values of initial data, using a MATLAB ODE45 numerical scheme.

\begin{tabular}{|c|c|c|c|c|c|c|c|c|}
\hline Example & $\begin{array}{c}\text { Initial } \\
\text { Data } \\
\text { (ID) }\end{array}$ & $\begin{array}{c}\text { Steady-state } \\
\text { solution (or } \\
\text { point) }\end{array}$ & $\lambda_{1}$ & $\lambda_{2}$ & $\lambda_{3}$ & $\lambda_{4}$ & $\lambda_{5}$ & TOS \\
\hline 1 & 1 & 1 & -18.1705 & -9.3804 & -5.7543 & -3.0150 & -4.0180 & Stable \\
\hline 2 & 2 & 2 & -18.1498 & -9.3527 & -5.6958 & -3.0150 & -4.0180 & Stable \\
\hline 3 & 3 & 3 & -18.1527 & -9.3547 & -5.7159 & -3.0150 & -4.0185 & Stable \\
\hline 4 & 4 & 4 & -18.1547 & -9.3568 & -5.7260 & -3.0150 & -4.0186 & Stable \\
\hline 5 & 5 & 5 & -18.1563 & -9.3589 & -5.7321 & -3.0150 & -4.0187 & Stable \\
\hline 6 & 6 & 6 & -18.1576 & -9.3607 & -5.7362 & -3.0150 & -4.0187 & Stable \\
\hline 7 & 7 & 7 & -18.1587 & -9.3623 & -5.7391 & -3.0150 & -4.0186 & Stable \\
\hline 8 & 8 & 8 & -18.1588 & -9.3621 & -5.7411 & -3.0150 & -4.0187 & Stable \\
\hline 9 & 9 & 9 & -18.1596 & -9.3633 & -5.7428 & -3.0150 & -4.0187 & Stable \\
\hline 10 & 10 & 10 & -18.1604 & -9.3644 & -5.7442 & -3.0150 & -4.0187 & Stable \\
\hline 11 & 11 & 11 & -18.1610 & -9.3654 & -5.7453 & -3.0150 & -4.0186 & Stable \\
\hline 12 & 12 & 12 & -18.1616 & -9.3664 & -5.7463 & -3.0150 & -4.0186 & Stable \\
\hline 13 & 13 & 13 & -18.1622 & -9.3672 & -5.7472 & -3.0150 & -4.0186 & Stable \\
\hline 14 & 14 & 14 & -18.1627 & -9.3680 & -5.7478 & -3.0150 & -4.0185 & Stable \\
\hline 15 & 15 & 15 & -18.1632 & -9.3688 & -5.7485 & -3.0150 & -4.0185 & Stable \\
\hline 16 & 16 & 16 & -18.1637 & -9.3695 & -5.7490 & -3.0150 & -4.0185 & Stable \\
\hline 17 & 17 & 17 & -18.1641 & -9.3701 & -5.7495 & -3.0150 & -4.0185 & Stable \\
\hline 18 & 18 & 18 & -18.1645 & -9.3708 & -5.7500 & -3.0150 & -4.0184 & Stable \\
\hline 19 & 19 & 19 & -18.1649 & -9.3714 & -5.7505 & -3.0150 & -4.0184 & Stable \\
\hline 20 & 20 & 20 & -18.1653 & -9.3720 & -5.7507 & -3.0150 & -4.0184 & Stable \\
\hline & & & & & & & & \\
\hline
\end{tabular}


where

Point $1=(25.4443,15.2308,30.7270,6.7195,2.1454)$,

Point $2=(25.3091,15.2308,30.6851,6.7195,2.1086)$,

Point $3=(25.3551,15.2308,30.6872,6.7195,2.1054)$,

Point $4=(25.3783,15.2308,30.6901,6.7195,2.1042)$,

Point $5=(25.3923,15.2308,30.6931,6.7195,2.1041)$,

Point $6=(25.4018,15.2308,30.6958,6.7195,2.1046)$,

Point $7=(25.4085,15.2308,30.6983,6.7195,2.1055)$,

Point $8=(25.4129,15.2308,30.6979,6.7195,2.1066)$,

Point $9=(25.4169,15.2308,30.6997,6.7195,2.1080)$,

Point $10=(25.4202,15.2308,30.7014,6.7195,2.1094)$,

Point $11=(25.4229,15.2308,30.7030,6.7195,2.1101)$,

Point $12=(25.4252,15.2308,30.7045,6.7195,2.1123)$,

Point $13=(25.4272,15.2308,30.7058,6.7195,2.1138)$,

Point $14=(25.4288,15.2308,30.7071,6.7195,2.1153)$,

Point $15=(25.4303,15.2308,30.7083,6.7195,2.1168)$,

Point $16=(25.4316,15.2308,30.7094,6.7195,2.1183)$,

Point $17=(25.4328,15.2308,30.7105,6.7195,2.1197)$,

Point $18=(25.4338,15.2308,30.7115,6.7195,2.1211)$,

Point $19=(25.4347,15.2308,30.7125,6.7195,2.1225)$,

Point $20=(25.4356,15.2308,30.7134,6.7195,2.1239)$.

What do we learn from Table 2? On the basis of this sophisticated computational approach which we have not seen elsewhere, we hereby infer that the stabilization of a five-dimensional dynamical system can be used as an alternative method of verifying qualitatively the concept of the stability of a unique positive steady-state solution which could have been a daunting task to resolve analytically.

However, this key contribution is only valid as long as the intrinsic growth rate $a_{1}$ is bigger than the intra-competition coefficient $a_{2}$ of the first competing species; the intrinsic growth rate $b_{1}$ is bigger than the intra-competition coefficient $b_{2}$ of the second competing species and the intrinsic growth rate of the resource biomass $c_{1}$ is bigger than the intra-competition coefficient $c_{2}$ of the resource biomass. In the event that the intra-competition coefficients of these three populations outweigh their corresponding intrinsic growth rates, will the specified steady-state solutions still be stable? Without loss of generality, it is interesting to observe that each of the twenty (20) stable steady-state solutions is also qualitatively well-defined within the choice of the model dynamics in the absence of proper model parameter estimation. The idea is consistent with the earlier discovery of Ekaka-a (2009).

\section{CONCLUSION AND RECOMMENDATION}

We have shown in this research that stabilization is an alternative way of testing for stability. Therefore, the application of a computational approach in the determination of the stability characteristic using the concept of stabilization is one of the contributions of this www.ijaems.com work that can be used to move the frontier of knowledge in the field of numerical mathematics with respect to stability of a dynamical system.We recommend a further investigation of the effect of fixed initial data for changing values of the independent variable.

\section{REFERENCES}

[1] Agarwal, M. and Devi, S. (2011). A resourcedependent competition model: Effects of toxicant emitted from external sources as well as formed by precursors of competing species. Nonlinear Analysis: Real World Application, 12, 751-766.

[2] Akpodee, R. E. and Ekaka-a, E. N. (2015). Deterministic stability analysis using a numerical simulation approach, Book of Proceedings - Academic Conference Publications and Research International on Sub-Sahara African Potentials in the new Millennium, 3(1).

[3] Chattopadhyay, J. (1996). Effects of toxic substances on a two-species competitive system, Ecological Model, 84, 287 - 289.

[4] Dhar, J., Chaudhary, M. and Sahu, G. P. (2013). Mathematical model of depletion of forestry resource, effect of synthetic-based industries. International Journal of Biological, Life Science and Engineering, $7,1-5$.

[5] De Luna, J. T. and Hallam, T. G. (1987). Effects of toxicants on populations: a qualitative approach IV. Resource-consumer-toxicant model, Ecological Model, 35, 249 - 273.

[6] Dubey, B. and Hussain, J. (2000). A model for allelopathic effect on two competing species, Ecological Model, 129, 195 - 207.

[7] Ekaka-a, E. N. (2009). Computational and mathematical modeling of plant species interactions in a harsh climate, Ph. D Thesis, University of Liverpool and University of Chester, United Kingdom.

[8] Freedman, H. I. and Shukla, J. B. (1991). Models for the effect of toxicant in single species and predator-prey systems, Journal of Mathematical Biology, 30, 15-30.

[9] Freedman, H. I. and So, J. W. H. (1985). Global stability and persistence of simple food chains, Mathematical Bioscience, 76, 69 - 86.

[10] Garcia-Montiel, D. C. and Scantena, F. N. (1994). The effects of human activity on the structure composition of a tropical forest in Puerto Rico, Forest Ecological Management, 63, 57 - 58.

[11] Hallam, T. G., Clark, C. E., and Jordan, G. S. (1983). Effects of toxicants on populations: a qualitative 
approach II. First order kinetics, Journal of Mathematical Biology, 18, $25-37$.

[12] Hallam, T. G., Clark, C. E., and Lassiter, R. R. (1983). Effects of toxicants on populations: a qualitative approach I. Equilibrium environmental exposure. Ecological Model, 18, 291-304.

[13] Hallam T. G. and De Luna, J. T. (1984). Effects of toxicants on populations: a qualitative approach III. Environmental and food chain pathways, Journal of Theoretical Biology, 109, $411-429$.

[14] Hsu, S. B., Li, Y. S. and Waltman, P. (2000). Competition in the presence of a lethal external inhibitor, Mathematical Bioscience, , 167, 177 - 199.

[15] Hsu, S. B. and Waltman, P (1998). Competition in the chemostat when one competitor produces a toxin, Japan Journal of Industrial and Applied Mathematics, 15,471-490.

[16] Huaping, L, and Zhien, M. (1991). The threshold of survival for system of two species in a polluted environment, Journal of Mathematical Biology, 30, 49 -61 .

[17]Lancaster, P. L. and Tismenetsky, M (1985).The Theory of Matrices, Second Edition, Academic Press, New York.

[18] Rescigno, A. (1977). The struggle for life - V. One species living in a limited environment, Bulleting of Mathematical Biology, 39, 479 - 485.

[19] Shukla, J. B., Agarwal, A., Dubey, B. and Sinha, P. (2001). Existence and survival of two competing species in a polluted environment: a mathematical model, Journal of Biological Systems, 9(2), 89 - 103.

[20] Shukla, J. B., Sharma, S., Dubey, B. and Sinha, P. (2009). Modeling the survival of a resource-dependent population: Effects of toxicants (pollutants) emitted from external sources as well as formed by its precursors, Nonlinear Analysis: Real World Application, 54-70.

[21] Thieme, H. R. (2000). Uniform persistence and permanence for non-autonomous semi-flows in population biology, Mathematical Bioscience, $166,173-201$.

[22] Yan, Y. and Ekaka-a, E. N. (2011). Stabilizing a mathematical model of population system, Journal of the Franklin Institute, 348(10), 2744 - 2758.

[23]Zhien, M. and Hallam, T. G. (1987). Effects of parameter fluctuations on community survival. Mathematical Bioscience, 86, 35 - 49. 